\title{
Sequential Optic Neuritis: A Neuromyelitis Optica Spectrum Disorder
}

\author{
Mehtab Alam¹, Mian Ayaz ul Haq¹, Asif Iqbal2, Kaleem Ullah² and Danish Nabi
}

\begin{abstract}
Neuromyelitis optica spectrum disorder (NMOSD) is an autoimmune relapsing demyelinating disorder which often leads to severe disability typically targeting spinal cord, optic nerves, and brainstem. Around $75 \%$ of NMOSD patients have serum immunoglobulin-G ( $\mathrm{lgG}$ ) autoantibodies to the aquaporin-4 channel (AQP4-lgG). AQP4-lgG antibodies have a central role in new diagnostic criteria of NMOSD. These antibodies have a critical role in long-term management after the first attack. The prevalence of this disorder is lower than multiple sclerosis in European countries. However, NMO makes a substantial proportion of the demyelinating diseases of the central nervous system in countries like Pakistan, where it can be mistaken for multiple sclerosis. Accurate diagnosis is essential as some of the drugs for multiple sclerosis can potentially worsen NMOSD. We present a case of sequential optic neuritis with positive aquaporin 4 antibodies. We have discussed the history, examination findings, diagnostic workups, and treatment of the patient.
\end{abstract}

Key Words: Neuromyelitis optica spectrum disorder, Devic's disease, Optic neuritis, Aquaporin 4, Alopecia areata.

\section{INTRODUCTION}

Neuromyelitis optica spectrum disorder (NMOSD) is an autoimmune disorder of central nervous system which presents with longitudinally extensive transverse myelitis (LETM), optic neuritis and area postrema syndrome (unexplained hiccups, nausea, and vomiting). ${ }^{1}$ The discovery of aquaporin-4 antibodies has led us to new diagnostic criteria and potential targets for new therapies. ${ }^{2}$ The authors are reporting the first-ever seropositive case of NMOSD in Pakistan. This case also points towards the association of NMOSD with alopecia areata, which has been described only once in the literature.

\section{CASE REPORT}

An 18-year girl patient presented with a 1-month history of sudden onset painful visual loss in her left eye. After two weeks, she had noticed decreased vision in her right eye as well. There was a past history of alopecia areata about 2 years ago, from which she made a complete recovery. She was also giving a history of intermittent headaches with migrainous features over the last four years. Examination showed no perception of light in the left eye and 6/36 acuity in the right eye. There were bilateral afferent pupillary defects (RAPD) with a constricted visual field in the right eye. Color vision was normal on the right side with no red desaturation. On fundoscopy, there was disc swelling, macular edema, splinter hemorrhages, retinal exudates and focal retinitis in the left eye. Similar but less severe findings were

Department of Neurologyl / Ophthalmology², Lady Reading

Hospital, Peshawar, Pakistan

Correspondence: Dr. Mehtab Alam, Department of Neurology,

Lady Reading Hospital, Peshawar, Pakistan

E-mail: mehtab39@gmail.com

Received: February 19, 2018; Accepted: October 24, 2018 noted in the right eye as well (Figure 1). The rest of her general physical and neurological examination was unremarkable.

Her baseline investigations were normal. ANA was negative. MRI brain with MRI orbit showed the prominence of fluid signals along both optic discs and optic nerves with enhancement of optic discs on postcontrast images (Figure 2). CSF showed normal cell count, protein, and glucose. There were unmatched oligoclonal bands (positive in CSF and negative in

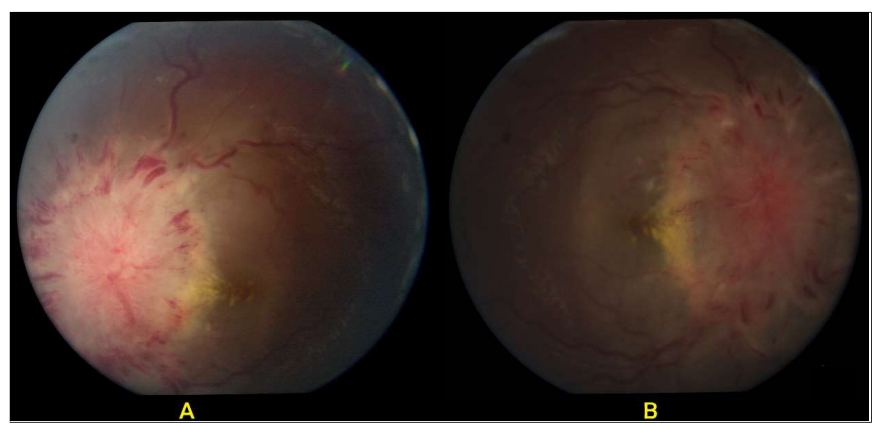

Figure 1: (A) Left fundus and (B) Right fundus at presentation showing bilateral $(B / L)$ disc swelling, macular star, splinter hemorrhages, retinal exudates and features focal retinitis.

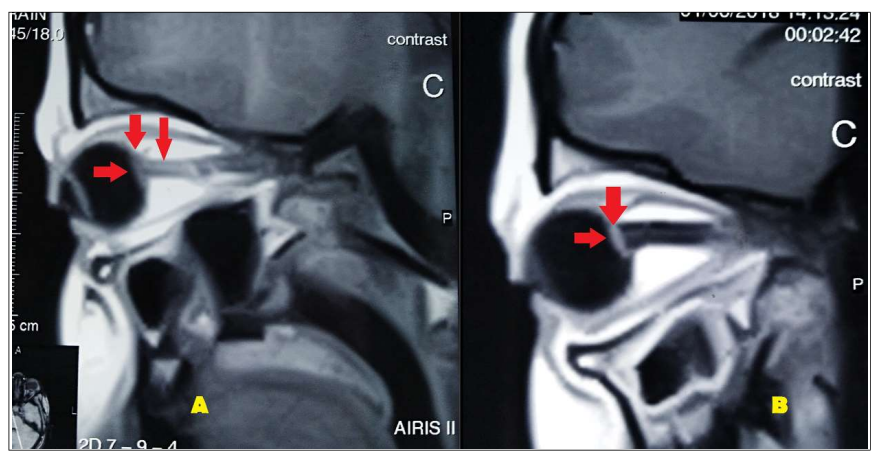

Figure 2: Post-contrast T1 images showing bilateral gadolinium-enhancing optic discs (right- A \& left -B) and right optic nerve. 
serum). Her serum APQ-4 IgG antibodies were positive and she was diagnosed as a case of NMOSD.

She was started on IV methylprednisolone $1 \mathrm{~g}$ for five days without any significant response. Therefore, we proceeded to plasma exchange. After five sessions of plasma exchange, she was commenced on oral prednisolone and azathioprine. Her vision has improved in 2 weeks with visual acuity of $6 / 9$ in the right eye and $1 / 60$ in the left eye. Fundoscopy findings also showed signs of settling disc edema along with optic disc pallor. The previously noticed peripapillary hemorrhages, retinal edema, and macular exudates had decreased. The dose of azathioprine has been increased and she remained on steroids. She was monitored closely in neurology and ophthalmology clinic.

\section{DISCUSSION}

Neuromyelitis optica spectrum disorder (NMOSD), formally known as Devic's disease, was once thought to be a variant of multiple sclerosis. The discovery of pathogenic APQ-4 IgG in 2004, not only led neurologists to classify it as a separate and distinct disease which typically targets spinal cord, optic nerve(s), area postrema, brainstem, and diencephalon; but also gave a base for the new International consensus diagnostic criteria for neuromyelitis optica spectrum disorders. 1,3

Going through the literature, we found three case reports of NMOSD from Pakistan, based on clinical and radiological features. ${ }^{4-6}$ NMOSD association with other autoimmune diseases has been reported in various cases and case series. To the best of authors' knowledge, so far only one case of NMOSD has been reported with concomitant Hashimoto thyroiditis and alopecia areata. ${ }^{7}$

The typical clinical manifestation of NMOSD is recurrent attacks of optic neuritis, which can be unilateral or bilateral and/or transverse myelitis with no/or partial recovery between attacks. NMOSD should be considered in LETM, which is defined as involvement of three or more than 3 consecutive vertebral segments on MRI. Additional clinical manifestations include intractable hiccups and/or nausea and vomiting (area postrema syndrome), brainstem syndrome, and symptomatic narcolepsy. During acute attacks, the neurologic deficits develop over days with an unpredictable recovery during subsequent months, causing progressive disability.2,8

Diagnosis of NMOSD is established, based on history, physical examination (looking for six core clinical characteristics proposed in IPND criteria for $\mathrm{NMOSD}^{3}$ ), and investigations. Depending on the mode of presentation, the initial evaluation of suspected NMOSD case may include ophthalmologic evaluation, APQ4-IgG status (preferably done on serum), MRI brain and spinal cord, and CSF analysis. It is equally important to exclude the mimics of NMOSD. The list of differential diagnosis of NMOSD is quite long, but the most important ones are multiple sclerosis (MS), acute disseminated encephalomyelitis (ADEM), idiopathic acute transverse myelitis, idiopathic optic neuritis, systemic autoimmune diseases (Sjogren's syndrome, systemic lupus erythematous, sarcoidosis), infective diseases (HIV, syphilis), and paraneoplastic causes. ${ }^{9}$ The differentiation of NMOSD from MS is crucial as some of the disease-modifying drugs approved for MS can worsen the NMOSD. ${ }^{2}$

The main aspects of management are the treatment of acute relapse, long-term therapy, and management of symptoms and rehabilitation. An acute attack is treated with 3-5 days course of IV corticosteroids (followed by oral steroids). Poor responders are treated with plasma exchange. Various medicine like azathioprine, methotrexate, mycophenolate etc. are used for long-term management (relapse prevention) as steroids sparing agents. If a patient relapse despite using these agents then rituximab is the next treatment option. 10 The new emerging and potential future therapeutic agents include eculizumab, anti-IL6 and anti-CD 19 monoclonal antibodies. ${ }^{2}$

The importance of APQ4-IgG status is that if a patient has only one episode (such as optic neuritis or acute myelitis) and seropositive for this antibody, that person will need relapse prevention therapy. ${ }^{10}$ After the availability of APQ-lgG assay in Pakistan, it is extremely important that the treating neurologist or physician is aware of this disorder and check this antibody in the relevant settings, as it has diagnostic as well as significant long-term treatment implications.

\section{REFERENCES}

1. Weinshenker BG, Wingerchuk DM. Neuromyelitis spectrum disorders. Mayo Clin Proc 2017; 92:663-79.

2. Whittam D, Wilson M, Hamid S, Keir G, Bhojak M, Jacob A. What's new in neuromyelitis optica? A short review for the clinical neurologist. J Neurol 2017; 264:2330-44.

3. Wingerchuk D, Banwell B, Bennett J, Cabre P, Carroll W, Chitnis $T$ et al. International consensus diagnostic criteria for neuromyelitis optica spectrum disorders. Neurology 2015; 85: 177-89.

4. Siddiqi SA, Hashmi M, Azmat Z, Mustafa S, Siddiqui KA. Pulmonary tuberculosis with neuromyelitis optica: An uncommon association of a common disease. J Coll Physicians Surg Pak 2012; 22:527-8.

5. Bhatty SA, Lal H, Talib A, Mahmood K, Naqvi I, Zaidi SS. Neuromyelitis optica (NMO) with abdominal tuberculosis (TB). $J$ Coll Physicians Surg Pak 2015; 25 (Suppl 2):S102-4.

6. Jan F, Shabir A, Ibrahim S. Neuromyelitis optica (devic's disease) in a 10 years old boy. Pak J Neurol Sci 2016; 11:48-51.

7. Yiannopoulou KG, Efthymiou AK, Karydakis KD. Neuromyelitis optica with coexisting autoimmune disorders. Arch Neurol 2008; 65:995.

8. Sahraian M, Radue E, Minagar A. Neuromyelitis optica, clinical manifestations and neuroimaging features. Neurol Clin 2013; 31:139-52.

9. Kim S, Kim S, Lee H, Kuroda H, Palace J, Fujihara K. Differential diagnosis of neuromyelitis optica spectrum disorders. Ther Adv Neurol Disord 2017; 10:265-89.

10. Palace J, Leite I, Jacob A. A practical guide to the treatment of neuromyelitis optica. Pract Neurol 2012; 12:209-14.

........... 